«Histoire de la Reine Berthe et du Roy Pepin». Mise en prose d'une chanson de geste. Edition critique par Piotr Tylus

\title{
Gianni Mombello
}

\section{(2) OpenEdition \\ Journals}

\section{Edizione digitale}

URL: https://journals.openedition.org/studifrancesi/39027

DOI: $10.4000 /$ studifrancesi.39027

ISSN: 2421-5856

\section{Editore}

Rosenberg \& Sellier

\section{Edizione cartacea}

Data di pubblicazione: 1 décembre 2004

Paginazione: 341-342

ISSN: 0039-2944

\section{Notizia bibliografica digitale}

Gianni Mombello, «Histoire de la Reine Berthe et du Roy Pepin». Mise en prose d"une chanson de geste. Edition critique par Piotr Tylus», Studi Francesi [Online], 143 (XLVIII | II) | 2004, online dal 30 novembre 2015, consultato il 19 mai 2021. URL: http://journals.openedition.org/studifrancesi/39027 ; DOI: https://doi.org/10.4000/studifrancesi.39027

Questo documento è stato generato automaticamente il 19 mai 2021.

\section{cc) (†) $\odot$}

Studi Francesi è distribuita con Licenza Creative Commons Attribuzione - Non commerciale - Non opere derivate 4.0 Internazionale. 


\title{
«Histoire de la Reine Berthe et du Roy Pepin». Mise en prose d'une chanson de geste. Edition critique par Piotr Tylus
}

\author{
Gianni Mombello
}

\section{NOTIZIA}

«Histoire de la Reine Berthe et du Roy Pepin». Mise en prose d'une chanson de geste. Edition critique par PIOTR TYLus, Genève, Droz, 2001 («Textes Littéraires Français», 536), pp. 348

Diciamo subito che si tratta di una eccellente edizione basata su di un "unicum» cartaceo dell'ultimo terzo del secolo XV, qua e là lacunoso che presenta difficoltà di lettura e soprattutto di interpretazione. La trascrizione è corredata da un buon lessico (pp. 307-40) e da un indice dei nomi propri (pp. 341-48).

2 L'acribia di P. Tylus si manifesta sopprattutto nell'ampia annotazione (pp. 263-306) nella quale dimostra un'ottima conoscenza del medio francese e consapevolezza delle innumeri insidie che testi di tale epoca presentano.

3 Si tratta di un'edizione conservativa, ma non aliena da interventi multipli, quando giudicati necessari ad una più fluida fruizione del testo. Se si può fare un appunto metodologico - che non è poi tale -, è il seguente. Il Curatore ha sempre cercato conferma dei suoi interventi nei dizionari specializzati (Godefroy, TL, FEW, Huguet) o in grammatiche storiche, partendo dalla non errata ma ideale supposizione che si tratti di repertori esaustivi. Ora non è il caso. Stimiamo che ci voglia un po' di coraggio per salvaguardare, in certi casi, caratteristiche, o stranezze (reali o supposte) di un testo anche quando non ricevono piena o almeno parziale conferma nei succitati strumenti di lavoro. Con prudenza, ovviamente, ma, in caso contrario, si resta al già noto e non vi è progresso nella ricerca. 
4 La redazione in prosa di Berte aus grans piés di Adenet le Roy sembra incoraggiare a correre dei rischi, diciamo bene dei rischi. A tal proposito, si può segnalare un «protexite»(riga 1280, i numeri fra parentesi rinvieranno, d'ora in poi, alle righe della presente edizione) che ha tutta l'aria di un «fantasma» («perplexité»?, parola dotta e recente - seconda metà del sec. XIV, e quindi mal letta?) e viene quindi rigettata in apparato senza sostituzione. Per contro, avremmo lasciato «tinderent»(1681) per «tindrent» (la forma con «d» epentetico era ancora accettata al tempo di Vaugelas e quella con la «e» potrebbe essere analogica con i verbi della quarta classe del tipo «vendre»- «vendierent» attestata. Cfr. FouchÉ, Le verbe, pp. 278, § 140 e p. 329, § 166), non saremmo intervenuti su «frenoise» (2208), che contiene l'esito fonologico di «-itia», anche se antico, ma forse duro a morire ( $\mathrm{Si}$ vedano, a tal proposito, le equilibrate riflessioni del Curatore a p. 57). Penso quindi che P. Tylus abbia fatto bene a conservare forme curiose, poco o per nulla attestate altrove come «et» per «est» $(53,3813,4086)$, «abreigee» (1175), «agectee»(1932), «fois» per «fais» (2153), «feust» per «fust» (2540), «vault» per «veult» (3072), «apareison» (3611), «s'esfray» (3733) per «s'esfraie», «voier» (4121) per «voir», «lauoit» (4370) per «looit», ecc. Si sarebbe potuto conservare anche «desire ay» (3025), malgrado la inabituale desinenza femminile (ma acettabile in un proparossitono) e forse anche «d'ecenssieurs» (4344) per «de censsiers»(encensoirs), ecc.

5 Un caso del tutto particolare è costituito dall'apparizione di una nasale dentale in sillaba iniziale di parole che contengono o no un altro simile fonema, ad esempio «consin» (1837), «prinzé» (3303), «antant»(4171, nota), modificata in «u» nel primo e terzo caso e mantenuta nel secondo. Scorrendo un incunabolo contenente i Ditz des sages hommes di Guillaume Tardif (1493 c.) ho trovato «sonvient», "sonvenance», «sonstenir», "gonverner», "gonverneurs» che sembrano confermare una tendenza, altrove ben attestata, della nasalizzazione - soprattutto della «o» - in sillaba iniziale, come appare in «monton» (mouton), «tronc de chou» (trous de chou"), «mont» (moult), «montepleier», «monche» (mouche), ecc. Questo fenomeno attende ancora di essere ben identificato, documentato e poi studiato poiché lo scambio fra «u» e «n» è molto frequente; tuttavia, esaminando attentamente buone edizioni quattro cinquecentesche, che distinguano accuratamente i due grafemi, si potrebbe precisare una tendenza ancora ignota del medio francese.

6 È quasi inutile aggiungere che gli interventi dell'Editore sono tutti misurati e spiegati (e. g: addizione di «bien»[1192], di «comme» [3089], ecc), perché facilitano la lettura, anche quando non sono strettamente necessari. Un caso particolarmente interessante è costituito dalla successione imperfetto-presente alle righe 3192-95 («Et qui demanderoit s'ilz y avoient de y venir de leurs propres motifs prins l'entreprinse ou par jeunesse qui aucunesfois les jeunes desiroyent et maine, respond l'istoire que non»). P. Tylus risolve riducendo alla terza del singolare del presente l'imperfetto «desiroyent», interrogandosi poi, nelle note, sul senso che potrebbe avere questo verbo in tale contesto (p. 295). La frase è contorta, ma «qui»(= «jeunesse») è soggetto di «maine» $\mathrm{e}$ non del verbo «desirer». Perché non lasciare «desiroyent» e far precedere «maine» da «[les]»?. Tuttavia, lo scontro fra questi due tempi stupisce, ma diventa più accettabile qualora si trattasse della concatenazione di due subordinate coordinate rette da una principale al presente (Adamas et Ydoine, vv. 4625-29: «Si com la route erroit tot droit / Et ils vienent a un destroit / [...] A destre uns chevaliers leur sourt». Cfr. P IMBS Propositions temporelles en ancien français. La détermination du moment, Paris, Les Belles 
Lettres, 1956, p. 281). Leggeremmo: «ou par jeunesse qui aucunesfois maine les jeunes gens et [qui la] desiroyent [= «desireroyent»?]». Concordiamo sul fatto che tale intervento è molto meno economico, ma potrebbe essere ridotto così: «ou par jeunesse qui aucunesfois les jeunes desiroyent et [(qui) les] maine».

7 Si potrebbe continuare a lungo ad analizzare il commento di questi interventi, tanto l'abilità del Curatore suscita, propone o risolve numerosi problemi linguistici legati al testo trascritto.

Bisogna tuttavia dire qualcosa anche a proposito dell'ottima introduzione, anche se in modo molto sintetico.

9 Schematizzata la storia recente del manosritto (da Berlino a Carcovia), descritto attentamente il materiale librario ed accennato ai suoi due antichi possessori: Jehanne Berruyere ed il marito Estienne Benard, vengono esaminati i lavori di Alfred Feist (1886), G. M. Dorsey (1933) e Annie Marez (1962), che hanno trascritto in parte o in toto il suo contenuto.

Ad un riassunto dell'opera, fa seguito un «état présent» degli studi su questa «mise en prose» dal quale si desume che essa dipende in massima parte della Berte aus grans piés di Adenet le Roy e non forse da un altro modello forse perduto (ipotesi Feist), con addizioni colte da altre fonti manoscritte o orali.

11 Molto impegnative sono le pagine seguenti sulla lingua del manoscritto di Cracovia leggermente piccardeggiante. Questa disamina si accentra innanzitutto sulle modifiche di contenuto e lessicali che producono una certa libertà di adattamento susseguente soprattutto al passaggio dal verso alla prosa, il che conduce ad amplificazioni o a riduzioni. Le modifiche di ordine stilistico sono ancora più rilevanti. Si notano, fra l'altro, la scomparsa di immagini e di stereotipi epici (ad esempio, la menzione dell'ulivo), le introduzioni e le chiuse epiche tipiche delle lasse, ecc. Se restano reminiscenze, come è ovvio, i cambiamenti di tono sono molti e traghettano questo testo dalla narrazione epica a quella romanzesca. L'ultimo paragrafo è dedicato ad altre redazioni che hanno come oggetto questa leggenda. Sono cinque e si tratta, per lo più, di cronache immaginarie, quella più vicina al nostro testo è il Miracle de Berthe che il prosatore avrebbe potuto conoscere. Si può lamentare che nulla sia detto della fortuna successiva di questo racconto che godette di una sopravvivenza notevole giunta fino a tempi molto a noi vicini. Non vi è nulla da aggiungere alle esaustive pagine che contengono i criteri adottati per la trascrizione.

12 Con questo libro, Piotr Tylus ci offre un solido apporto ad una migliore conoscenza dell'evoluzione della prosa francese quattrocentesca forse frutto dell'attività di uno scrittore attivo in ambito borgognone se non proprio alla corte del Grand Duca d'Occidente. 